\title{
Influence of Fluctuating Circulations on the Transport Phenomena from Cylinders Including Uniform Suction or Blowing
}

\author{
Wen-Jei Yang* and Tomoaki IzUmi $\dagger$ \\ University of Michigan, Ann Arbor, Mich.
}

\begin{abstract}
This paper presents a theoretical investigation of the influence of fluctuating circulation (flow with magnitude- and direction-dependent amplitudes) upon the transfer of momentum, heat, and mass in two-dimensional laminar boundary-layer fow past cylinders with or without uniform suction. The boundary-layer equations for flow, temperature, and concentration are linearized by use of a perturbation technique. The solutions of the velocity, temperature, and concentration components are obtained by power-series development so that the universal distribution functions may be applied to any two-dimensional fow. Theoretical results include the frequency response of fluid velocity, temperature, and concentration, the streamline patterns of the steady streaming, the distribution of the steady second-order temperature and concentration, and the net variations in the shear stress, rates of heat, and mass transfer. Numerical results are obtained for flows around a circular cylinder with fluctuating circulations of constant and space-dependent amplitudes.
\end{abstract}

\section{Nomenclature}

$=$ coefficient depending on the geometrical configuration of the body, dimensionless

= coefficient depending on the nature of flow oscillation, dimensionless

$c \quad=$ dimensionless concentration; $=\left(C_{w}{ }^{*}-C^{*}\right) /\left(C_{w}{ }^{*}-\right.$ $\left.C_{\infty}{ }^{*}\right)$

$C^{*} \quad=$ concentration, lbm-mole; $C_{w}{ }^{*}$ at the wall; $C_{\infty}{ }^{*}$ of the freestream

$F \quad=$ functional coefficient or universal distribution function of temperature (or concentration), dimensionless: $F_{0 k}$ for the zeroth-order approximation; $F_{1 l}$ for the first-order approximation; $F_{2 l i k}$ for the second-order approximation

= functional coefficient or universal distribution function of velocity, dimensionless; $f_{0 k}$ for the zerothorder approximation; $f_{1 l k}$ for the first-order approximation; $f_{2 l j k}$ for the second-ordera pproximation

$=(-1)^{1 / 2}$

$=$ integer, dimensionless

$=$ characteristic length, $\mathrm{ft} ;=2 R$ for a circular cylinder

$=$ integer, dimensionless

$=$ constant, dimensionless

$N u=$ Nusselt number, dimensionless; $=(\partial \theta / \partial y)_{y=0}$

$=$ constant, dimensionless

$=$ Prandtl number, dimensionless

$=$ rate of heat transfer, $\mathrm{Btu} / \mathrm{hr}-\mathrm{ft}^{2}$

$=$ radius of a circular cylinder, $\mathrm{ft}$

$=$ Reynolds number, dimensionless

$=$ Schmidt number, dimensionless

$=$ Sherwood number, dimensionless; $=(\partial c / \partial y)_{y=0}$

$=$ dimensionless temperature $;=\left(T_{w}{ }^{*}-T^{*}\right) /\left(T_{w}{ }^{*}-\right.$ $\left.T_{\infty}^{*}\right)$

$T^{*} \quad=$ temperature, ${ }^{\circ} \mathrm{F} ; T_{w}{ }^{*}$ of the wall; $T_{\infty}{ }^{*}$ of the freestream

$t \quad=$ dimensionless time; $=t^{*} U_{\infty} / L$

$t^{*} \quad=$ physical time, $\mathrm{hr}$

$U(x, t)=$ velocity of potential flow in dimensionless form; $=U^{*}(x, t) / U_{\infty}$

$U_{0}(x)=$ time-average velocity of potential flow in dimensionless form; $=U_{0}^{*}(x) / U_{\infty}$

$U_{1}(x)=$ oscillation amplitude of potential flow in dimensionless form; $=U_{1}^{*}(x) / U_{\infty}$

Received June 26, 1964; revision received November 25, 1964. This work is a publication from the Heat Transfer Laboratory, Department of Mechanical Engineering, University of Michigan. The authors wish to express their appreciation to the Computing Center at the university for providing the IBM 7090 digital computer for the numerical computations.

* Assistant Professor of Mechanical Engineering.

$\uparrow$ Graduate Student Assistant of Mechanical Engineering.
$U_{\infty} \quad=$ velocity of potential flow at infinity, $\mathrm{ft} / \mathrm{hr}$

$U^{*}(x, t)=$ velocity of potential flow; $=U_{0}^{*}(x)+\epsilon_{1}^{*}(x)$ $\cos \omega t, \mathrm{ft} / \mathrm{hr}$

$U_{0}^{*}(x)=$ time-average velocity of potential flow, $\mathrm{ft} / \mathrm{hr}$

$U_{1}^{*}(x)=$ oscillation amplitude of potential flow, $\mathrm{ft} / \mathrm{hr}$

$u \quad=$ dimensionless velocity in $x$ direction, $=u^{*} / u_{\infty} ; u_{0}$ for the zeroth-order perturbation, $=\partial \psi_{0} / \partial y ; u_{1}$ and $u_{1 l}$ for the first-order perturbation, $u_{1 l}=$ $\partial \psi_{12} / \partial y ; u_{2}$ and $u_{2 l i}$ for the second-order perturbation, $u_{2 l j}=\partial \psi_{2 l j} / \partial y$

$u^{*} \quad=$ velocity component in $x$ direction, $\mathrm{ft} / \mathrm{hr}$

$V=$ velocity of uniform suction in dimensionless form; $=V^{*} / U_{\infty}$

$V^{*} \quad=$ velocity of uniform suction, $\mathrm{ft} / \mathrm{hr}$

$v \quad=$ dimensionless velocity in $y$ direction, $=v^{*} / U_{\infty} ; v_{0}$ for the zeroth-order perturbation, $=-\partial \psi_{0} / \partial x ; v_{1}$ and $v_{1 l}$ for the first-order perturbation, $v_{1 l}=$ $-\partial \psi_{1 l} / \partial x ; v_{2}$ and $v_{2 l i}$ for the second-order perturbation, $v_{2 l i}=-\partial \psi_{2 l i} / \partial x$

$v^{*} \quad=$ velocity component in $y$ direction, $\mathrm{ft} / \mathrm{hr}$

$x \quad=$ distance measured along the wall in dimensionless form; $=x^{*} / L$

$x^{*} \quad=$ distance measured along the wall, $\mathrm{ft}$

$y=$ dimensionless distance measured in the direction perpendicular to the wall; $=y^{*} / L$

$y^{*} \quad=$ distance measured in the direction perpendicular to the wall, $\mathrm{ft}$

$=$ small constant parameter, dimensionless

$=$ dimensionless distance measured in the direction perpendicular to the wall; $y\left(a_{1} R e\right)^{1 / 2}$

$=$ dimensionless temperature; $\theta_{0}$ for the zeroth-order perturbation $=T_{0} ; \theta_{1 l}$ for the first-order perturbation; $\theta_{2 l i}$ for the second-order perturbation

$\mu \quad=$ absolute viscosity, $\mathrm{lbm} / \mathrm{hr}-\mathrm{ft}$

$\tau \quad=$ wall shear stress in dimensionless form; $=\tau^{*} L / \mu U_{\infty}$ or $=(\partial u / \partial y)_{y=0}$

$\tau^{*} \quad=$ wall shear stress; $=-\mu\left(\partial u^{*} / \partial y^{*}\right)_{y=0}, \mathrm{lbf} / \mathrm{ft}^{2}$

$\Psi=$ Stokes stream function in dimensionless form, $=\psi^{*} / L U_{\infty} ; \mathbf{\Psi}_{0}$ for the zeroth-order perturbation, $=\Psi_{0}^{*} / L U_{\infty} ; \Psi_{1 l}$ for the first-order perturbation $=\Psi_{1 l}{ }^{*} U_{\infty}{ }^{l-1} / L^{l+1} ; \Psi_{2 l j}$ for the second-order perturbation $=\Psi_{2 l j}{ }^{*} U_{\infty}{ }^{l-1} / L^{l+1}$

$\Psi^{*} \quad=$ Stokes stream function, $\mathrm{ft}^{2} / \mathrm{hr} ; \Psi^{*}$ for the zerothorder perturbation; $\Psi_{1 l^{*}}$ for the first-order perturbation; $\Psi_{2 l \jmath k^{*}}$ for the second-order perturbation

$\omega=$ frequency of oscillation in dimensionless form; $=\omega^{*} L / U_{\infty}$

$\omega^{*} \quad=$ frequency of oscillation, $\mathrm{rad} / \mathrm{hr}$

\section{Superscripts}

()$^{\prime},()^{\prime \prime},()^{\prime \prime \prime}=$ first, second, and third derivatives with respect to $\eta$, respectively 


\section{Subscripts for functional coefficients $f$ and $F$}

$0,1,2=$ zeroth-, first-, and second-order perturbation

$j=$ either steady- or transient-state in the second-order perturbation

$l=$ order of approximation in terms of the frequency of oscillation, $(i \omega)^{2}$

$k \quad=$ order of function

$s=$ time-independent component in the second-order perturbation

$t \quad=$ time-dependent component in the second-order perturbation

\section{Introduction}

$\mathbf{R}$ ECENTLY much work has been published on the theory of laminar boundary layers in oscillatory motions caused by the vibrations of solid surfaces or flow oscillations. ${ }^{1-19}$ A comprehensive review of the literature is presented in Ref. 21 and will not be repeated here.

It is generally observed that a steady flow, known as secondary flow, exists in an oscillating fluid or is generated in a quiescent fluid where solid boundaries oscillate. This phenomenon is also known to occur when oscillating acoustic waves interact with a stationary object.

This paper is devoted to the study of the effects of fluctuating circulation (or flow oscillation with magnitude- and direction-dependent amplitudes) on the transport phenomena in two-dimensional laminar boundary-layer flow past cylinders. The only perturbing force is that due to the flow circulation with a small fluctuating amplitude and low frequency in an otherwise forced convective field. One example is a uniform flow about a heated bluff body with a trailing row of alternating vortices. This induces oscillations in transport phenomena.

To solve the transport equations for low frequencies, Lighthill ${ }^{1}$ used a Karman-Pohlhausen method and Hori ${ }^{3}$ used the method of Blasius and Howarth. At high frequencies, where viscosity is only effective for oscillation within a very thin shear-wave boundary layer close to the wall, the theory of differential equations with a large parameter was applied independently by Lighthill ${ }^{1}$ and Lin. ${ }^{2}$ For the present investigation, the transfer equations are solved by power-series development from the stagnation point, without any arbitrary assumptions regarding the velocity, temperature, and concentration profiles. The solutions expressed in terms of the coefficients representing the geometrical configuration, the nature of the fluctuating circulations, and the universal distribution functions may therefore be applied to any twodimensional flow.

The effects of the uniform suction and blowing are also treated. Theoretical analyses include the frequency response of velocity, temperature, and concentration, the alterations in the wall shear stress and the rates of heat and mass transfer, the streamline pattern of the streaming motion, and the distribution of the steady second-order temperature and concentration. Numerical results are obtained for the flow past a circular cylinder with fluctuating circulations having the constant and space-dependent amplitudes.

\section{Fundamental Equations}

The physical system consists of a heated cylindrical body subjected to a transverse flow that fluctuates harmonically with time. A coordinate system $x^{*}, y^{*}$ is fixed at the forward stagnation point, with $x^{*}$ measured along the cylindrical surface and $y^{*}$ in the direction perpendicular to the surface. The analysis is restricted to two-dimensional, incompressible flow in the $x^{*}-y^{*}$ plane. The external potential flow is represented by $U^{*}\left(x^{*}, t^{*}\right)=U_{0}^{*}\left(x^{*}\right)+\epsilon U_{1}^{*}\left(x^{*}\right) \cos \omega^{*} t^{*}$, where $U_{0}^{*}(x)$ is the time-average velocity, $\epsilon U_{1}^{*}(x)$ is the amplitude of oscillation, $\omega^{*}$ is the frequency of oscillation, and $t^{*}$ is the physical time.
The cylinder with surface concentration of $C_{w} *$ is heated to a uniform temperature $T_{w}{ }^{*}$. It is maintained in contact with a fluid at temperature $T_{\infty} *$, and the concentration $C_{\infty}{ }^{*}$, which otherwise would flow with a constant velocity $U_{\infty}$ at infinity.

The following assumptions are imposed on the analysis: 1) the velocity components are small compared to sonic velocity, so that the compressibility effects are negligible; 2) in the temperature boundary layer, the viscous dissipation and the heat generated by change in pressure may be neglected; 3 ) the differences in temperature and concentration are not so large that the physical properties of the fluid do not vary from point to point; and 4) for problems involving suction or blowing, the flow through the surface is assumed to be wholly normal, since the pressure gradient through the surface is usually large. With these assumptions, the boundary-layer equations for flow, temperature, and concentration reduce to

$$
\begin{gathered}
\frac{\partial u}{\partial x}+\frac{\partial v}{\partial y}=0 \\
\frac{\partial u}{\partial t}+u \frac{\partial u}{\partial x}+v \frac{\partial u}{\partial y}=\frac{\partial U}{\partial t}+U \frac{\partial U}{\partial x}+\frac{1}{R e} \frac{\partial^{2} u}{\partial y^{2}} \\
\frac{\partial T}{\partial t}+u \frac{\partial T}{\partial x}+v \frac{\partial T}{\partial y}=\frac{1}{R e P r} \frac{\partial^{2} T}{\partial y^{2}} \\
\frac{\partial C}{\partial t}+u \frac{\partial C}{\partial x}+v \frac{\partial C}{\partial y}=\frac{1}{R e S c} \frac{\partial^{2} C}{\partial y^{2}} \\
y=0: u=T=C=0 \\
y\left\{\begin{array}{l}
0 \text { for no suction or blowing } \\
V\left\{\begin{array}{l}
\text { positive for uniform blowing } \\
\text { negative for uniform suction }
\end{array}\right.
\end{array}\right.
\end{gathered}
$$

where the external potential flow is $U(x, t)=U_{0}(x)+\epsilon U_{1}(x)$ coswt. Because of the identical form of the equations as well as boundary conditions for temperature and concentration, both may be treated simultaneously. In the following analysis one may therefore interchange the quantities $T$ and $C$, $P r$ and $S c$, and $N u$ and $S h$.

\section{Solutions}

Considering the nature of the velocity of the potential flow $U(x, t)$, which is imposed as perturbations, the following forms may be assumed for the function involved:

$$
\left.\begin{array}{l}
u(x, y, t)=u_{0}(x, y)+\epsilon u_{1}(x, y, t)+\epsilon^{2} u_{2}(x, y, t)+\ldots \\
v(x, y, t)=v_{0}(x, y)+\epsilon v_{1}(x, y, t)+\epsilon^{2} v_{2}(x, y, t)+\ldots \\
T(x, y, t)=T_{0}(x, y)+\epsilon T_{1}(x, y, t)+\epsilon^{2} T_{2}(x, y, t)+\ldots
\end{array}\right\}
$$

By substituting these expansions into the governing equations and boundary conditions, and by separating terms according to the powers of $\epsilon^{n}$, a set of simultaneous, linear differential equations and boundary conditions are found as follows:

Zeroth-Order Perturbation, $\epsilon^{0}$

$$
\begin{gathered}
\frac{\partial u_{0}}{\partial x}+\frac{\partial v_{0}}{\partial y}=0 \\
u_{0} \frac{\partial u_{0}}{\partial x}+v_{0} \frac{\partial u_{0}}{\partial y}=\frac{1}{R e} \frac{\partial^{2} u_{0}}{\partial y^{2}}+U_{0} \frac{d U_{0}}{d x} \\
u_{0} \frac{\partial T_{0}}{\partial x}+v_{0} \frac{\partial T_{0}}{\partial y}=\frac{1}{R e P r} \frac{\partial^{2} T_{0}}{\partial y^{2}} \\
v_{0}=\left\{\begin{array}{l}
y=0: u_{0}=T_{0}=0 \\
v\left\{\begin{array}{l}
\text { positive for uniform blowing } \\
\text { negative for uniform suction }
\end{array}\right.
\end{array}\right.
\end{gathered}
$$


Table 1 Subseripts for universal functions $f$ and $F$

Subscript

Order of

Perturbation

1st

2nd

3rd

4 th

5 th

\begin{tabular}{lllll}
$\begin{array}{l}\text { Zeroth } \\
\text { 1st }\end{array}$ & Order of $\epsilon$ & Exponent of $x$ & Order of function & $\ldots$ \\
2 nd & Order of $\epsilon$ & Order of $\omega$ & Exponent of $x$ & Order of function \\
Order of $\epsilon$ & Order of $\omega$ & Steady or transient state & Exponent of $x$ & Order of function \\
\hline
\end{tabular}

First-Order Perturbation, $\epsilon^{1}$

Second-Order Perturbation, $\epsilon^{2}$

$$
\frac{\partial u_{1}}{\partial x}+\frac{\partial v_{1}}{\partial y}=0
$$

$\frac{\partial u_{1}}{\partial t}+u_{\mathrm{I}} \frac{\partial u_{0}}{\partial x}+u_{0} \frac{\partial u_{1}}{\partial x}+v_{0} \frac{\partial u_{1}}{\partial y}+v_{1} \frac{\partial u_{0}}{\partial y}=$

$$
\left(U_{1} \frac{d U_{0}}{d x}+U_{0} \frac{d U_{1}}{d x}\right) \cos \omega t-\omega U_{1} \sin \omega t
$$

$\frac{\partial T_{1}}{\partial t}+u_{0} \frac{\partial T_{1}}{\partial x}+u_{1} \frac{\partial T_{0}}{\partial x}+v_{0} \frac{\partial T_{1}}{\partial y}+$

$$
\begin{gathered}
v_{1} \frac{\partial T_{0}}{\partial y}=\frac{1}{\operatorname{RePr}} \frac{\partial^{2} T_{1}}{\partial y^{2}} \\
y=0: u_{1}=v_{1}=T_{1}=0 \\
y=\infty: u_{1}=U_{1}(x) \cos \omega t, \quad T_{1}=0
\end{gathered}
$$

\begin{tabular}{|c|c|c|c|c|c|}
\hline Tunction & Differential equations & & $\eta=0$ & $\eta=$ & $=\infty$ \\
\hline$f_{01}$ & $=\left(f_{01}{ }^{\prime}\right)^{2}-f_{01} \cdot f_{01}{ }^{\prime \prime}-1$ & $f_{01}$ & $=\left\{\begin{array}{l}0 \\
V\end{array} f_{01}^{\prime}=0\right.$ & $f_{01}^{\prime}$ & $=$ \\
\hline$f_{03}$ & $f_{03}^{\prime \prime \prime}=4 f_{01}{ }^{\prime} \cdot f_{03}^{\prime}-3 f_{01}^{\prime \prime} \cdot f_{03}-f_{01} \cdot f_{03}-1$ & $f_{03}$ & $=f_{03}^{\prime}=0$ & $f_{03}^{\prime}$ & \\
\hline$f_{051}$ & $f_{051}^{\prime \prime \prime}=6 f_{01}{ }^{\prime} \cdot f_{051}{ }^{\prime}-5 f_{01}^{\prime \prime} \cdot f_{051}-f_{01} \cdot f_{051}{ }^{\prime \prime}-1$ & $f_{0 \text { ă1 }}$ & $=f_{051}^{\prime}=\frac{1}{6}$ & $f_{051}^{\prime}$ & \\
\hline$f_{052}$ & $f_{052}^{\prime \prime \prime}=f_{01}^{\prime} \cdot f_{052}{ }^{\prime}+8\left(f_{04}^{\prime}\right)^{2}-5 f_{01}{ }^{\prime \prime} f_{052}-8 f_{04} \cdot f_{04}{ }^{\prime \prime}-f_{01} \cdot f_{052}{ }^{\prime \prime}-0.5$ & $f_{052}$ & $=f_{052}^{\prime}=0$ & $f_{052}$ & $=$ \\
\hline$f_{100}$ & $f_{100}^{\prime \prime \prime}=f_{01}^{\prime} \cdot f_{100}^{\prime}-f_{61} \cdot f_{100}^{\prime \prime}-1$ & $f_{100}$ & $=f_{100^{\prime}}=0$ & $f_{100}^{\prime}$ & $=$ \\
\hline$f_{1021}$ & $f_{1021}^{\prime \prime \prime}=3 f_{01}^{\prime} \cdot f_{1021}^{\prime}-2 f_{01}^{\prime \prime} \cdot f_{1021}-f_{01} \cdot f_{1021}^{\prime \prime}-1$ & $f_{1021}$ & $=f_{1021}{ }^{\prime}=0$ & $f_{1021}{ }^{\prime}$ & $=$ \\
\hline$f_{1022}$ & $f_{1022}^{\prime \prime \prime}=3 f_{01}^{\prime} \cdot f_{1022}{ }^{\prime}-2 f_{01}^{\prime \prime} \cdot f_{1022}-f_{01} f_{1022}^{\prime \prime}-1+4\left(f_{03}^{\prime} \cdot f_{100}^{\prime}-f_{03} f_{100}{ }^{\prime \prime}\right)$ & $f_{1022}$ & $=f_{1022}{ }^{\prime}=0$ & $f_{1022}{ }^{\prime}$ & $=$ \\
\hline$f_{1041}$ & $f_{1041}^{\prime \prime \prime}=5 f_{01}^{\prime} \cdot f_{1041}^{\prime}-f_{01}^{\prime} f_{1041}^{\prime \prime}-4 f_{01}^{\prime \prime} f_{1041}-1$ & $f_{1041}$ & $=f_{1041^{\prime}}=0$ & $f_{1041^{\prime}}$ & $=$ \\
\hline$f_{1042}$ & $f_{1042}^{\prime \prime \prime}=5 f_{01}^{\prime} f_{1042}+12 f_{04}^{\prime} f_{1021}-f_{01} f_{1042}{ }^{\prime \prime}-7.2 f_{04} \cdot f_{1021}^{\prime \prime}-4 f_{01}^{\prime \prime} f_{1042}-4.8 f_{04}^{\prime \prime} f_{1021}-1$ & $f_{1042}$ & $=f_{1042}{ }^{\prime}=0$ & $f_{1042}{ }^{\prime}$ & $=$ \\
\hline 043 & $f_{1043}{ }^{\prime \prime \prime}=5 f_{01}^{\prime} f_{1043}+6 f_{100} f_{051}^{\prime}-f_{01} f_{1043}-4 f_{01}{ }^{\prime \prime} f_{1043}-1$ & $f_{1043}$ & $=f_{1043}{ }^{\prime}=0$ & $f_{1043^{\prime}}$ & $=$ \\
\hline 1044 & $\begin{array}{c}f_{1044}{ }^{\prime \prime \prime}=5 f_{01}^{\prime} f_{1044}+12 f_{04}^{\prime} f_{1032}+6 f_{100} f_{052}^{\prime}-f_{01} f_{1044}-7.2 f_{04} f_{1022}^{\prime \prime}-4 f_{01}^{\prime \prime} f_{1044} \\
-4.8 f_{04}^{\prime \prime} f_{1022}\end{array}$ & $f_{1044}$ & $=f_{1044}{ }^{\prime}=0$ & $f_{1044}^{\prime}$ & $=$ \\
\hline$f_{20 s 11}$ & $f_{20 s 11}{ }^{\prime \prime \prime}=2 f_{01}^{\prime} f_{20 s 11}^{\prime}+2 f_{100}^{\prime} f_{1021}^{\prime}-f_{01} f_{20 s 11}^{\prime \prime}+2 f_{100}^{\prime \prime} f_{1021}-f_{01}^{\prime \prime} f_{20 s 11}-0.666$ & $f_{20 s 11}$ & $=f_{20 s+1}^{\prime}=0$ & $f_{20: 11}$ & $=0$ \\
\hline$f_{20 s 12}$ & $f_{20 s 12}{ }^{\prime \prime \prime}=2 f_{01}^{\prime} f_{20 s 12}{ }^{\prime}+2 f_{100}^{\prime} f_{1022}^{\prime}-f_{01} f_{20 s 12}^{\prime \prime}+2 f_{100}^{\prime \prime} f_{1022}-f_{01}^{\prime \prime} f_{20 s 12}$ & $f_{20 s 12}$ & $=f_{20 s 12}{ }^{\prime}=0$ & $f_{20 s 12}^{\prime}$ & $=$ \\
\hline$f_{20833}$ & $\begin{array}{c}f_{20833}{ }^{\prime \prime}=8 f_{04}{ }^{\prime} f_{20 s 11}{ }^{\prime}+4 f_{01}^{\prime} f_{20 s 33}+3.333 f_{100} f_{1042}^{\prime}+6 f_{1021} f_{1022}^{\prime}-f_{01} f_{20 s 33}-6 f_{04} f_{20 s 11} \\
-3 f_{1021} f_{1022}^{\prime \prime}-3 f_{100}^{\prime \prime} f_{1022}-3.333 f_{100}{ }^{\prime \prime} f_{1042}-2 f_{04}{ }^{\prime \prime} f_{20 s 11}-3 f_{01}{ }^{\prime \prime} f_{20 s 33}\end{array}$ & $f_{20 s 33}$ & $=f_{20 s 33}{ }^{\prime}=0$ & $f_{20 s 33}{ }^{\prime}$ & $=$ \\
\hline$f_{20 \pi 34}$ & $\begin{array}{c}f_{20834}{ }^{\prime \prime \prime}=8 f_{04}^{\prime} f_{20 s 12}+4 f_{01}^{\prime} f_{20 s 34}+3.333 f_{100} f_{1044}+3\left(f_{1022}{ }^{\prime}\right)^{2}-f_{01} f_{20834}{ }^{\prime \prime}-6 f_{04} f_{20 s 12}{ }^{\prime \prime} \\
-3 f_{1022}{ }^{\prime \prime} f_{1022}-3.333 f_{100}{ }^{\prime \prime} f_{1044}-2 f_{04}{ }^{\prime \prime} f_{20812}-3 f_{01}^{\prime \prime} f_{20834}\end{array}$ & $f_{20834}$ & $=f_{20 s ; 4}{ }^{\prime}=0$ & $f_{20 s i 4}{ }^{\prime}$ & $=$ \\
\hline$f_{20835}$ & $f_{20 s 85}^{\prime \prime \prime}=4 f_{01}^{\prime} f_{20 s 35}+3.333 f_{100}^{\prime} f_{1043}^{\prime}-f_{01} f_{20 s 35}-3.333 f_{100}^{\prime \prime} f_{1043}-3 f_{01}^{\prime \prime} f_{20 s 35}$ & $f_{20 s 35}$ & $=f_{20 s 35}{ }^{\prime}=0$ & $f_{20835}{ }^{\prime}$ & $=$ \\
\hline$F_{00}$ & $=-P_{r} f_{01} F_{00}^{\prime}$ & $F_{00}$ & $=0$ & $F_{00}$ & \\
\hline$F_{02}$ & $=P_{r}\left(2.0 f_{01}^{\prime} F_{02}-3.0 f_{04} F_{00}^{\prime}-f_{01} F_{02}^{\prime}\right)$ & $F_{02}$ & $=0$ & $F_{02}$ & $=0$ \\
\hline$F_{041}$ & $=P_{r}\left(-f_{01} F_{041}^{\prime}+4 f_{01}^{\prime} F_{041}-5 f_{051} F_{00}^{\prime}\right)$ & $F_{041}$ & $=0$ & $F_{041}$ & $=0$ \\
\hline$F_{042}$ & $F_{042}^{\prime \prime}=P_{r}\left[-f_{01} F_{042}^{\prime}+4 f_{01}^{\prime} F_{042}-5 f_{052} F_{00}^{\prime}+\frac{8}{3}\left(2 f_{04}^{\prime} F_{02}-3 f_{04} F_{02}^{\prime}\right)\right]$ & $F_{042}$ & $=0$ & $F_{042}$ & $=0$ \\
\hline$F_{1011}$ & $F_{1011}^{\prime \prime}=P_{r}\left(f_{01}^{\prime} F_{1011}-f_{01} F_{1011}^{\prime}-3.0 f_{102} F_{00}^{\prime}\right)$ & $F_{1011}$ & $=0$ & $F_{1011}$ & $=0$ \\
\hline$F_{1012}$ & $F_{1012}{ }^{\prime \prime}=P_{r}\left(f_{01}^{\prime} F_{1012}+4.0 f_{100}{ }^{\prime} F_{02}-f_{01} F_{1012}^{\prime}-3.0 f_{104} F_{00}^{\prime}\right)$ & $F_{1012}$ & $=0$ & $F_{1012}$ & $=$ \\
\hline$F_{1031}$ & $F_{1031}^{\prime \prime}=P_{r}\left(3 f_{01}^{\prime} F_{1031}-f_{01} F_{1031}^{\prime}-5 F_{00}^{\prime} f_{1041}\right)$ & $F_{1031}$ & $=0$ & $F_{1031}$ & $=0$ \\
\hline$F_{1032}$ & $\begin{array}{c}F_{1032}{ }^{\prime \prime}=P_{r}\left(2 f_{04}{ }^{\prime} F_{1011}+3 f_{01}{ }^{\prime} F_{1032}+6 F_{02} f_{1021}{ }^{\prime}-6 f_{04} F_{1011}{ }^{\prime}-f_{01} F_{1032}{ }^{\prime}-6 f_{1021} F_{02}{ }^{\prime}\right. \\
\left.\quad-5 f_{1042} F_{00}^{\prime}\right)\end{array}$ & $F_{1022}$ & $=0$ & $F_{1032}$ & \\
\hline$F_{1033}$ & $F_{1083}{ }^{\prime \prime}=P_{r}\left(3 f_{01}^{\prime} F_{1033}+6 f_{100}^{\prime} F_{041}-f_{01} F_{1033}^{\prime}-5 f_{1043} F_{00}^{\prime}\right)$ & $F_{1033}$ & $=0$ & $F_{1033}$ & \\
\hline$F_{1034}$ & $\begin{aligned} F_{1034}{ }^{\prime \prime}= & P_{r}\left(2 f_{04}{ }^{\prime} F_{1012}+3 f_{01}{ }^{\prime} F_{1034}+6 f_{100} F_{042}+6 f_{1022} F_{02}-6 f_{04} F_{1012}{ }^{\prime}-f_{01} F_{1034}{ }^{\prime}\right. \\
& \left.-6 F_{02}^{\prime} f_{1022}-5 f_{1044} F_{06}^{\prime}\right)\end{aligned}$ & $F_{1034}$ & $=0$ & $F_{1034}$ & \\
\hline$F_{20 s 01}$ & $F_{20 s 01}{ }^{\prime \prime}=P_{r}\left(0.666 f_{100} F_{1011}-f_{01} F_{20 s 01}^{\prime}-f_{20 s 11} F_{00}{ }^{\prime}\right)$ & $F_{\mathrm{z} 0 \mathrm{~s} 01}$ & $=0$ & $F_{20 s 01}$ & \\
\hline$F_{20 s 02}$ & 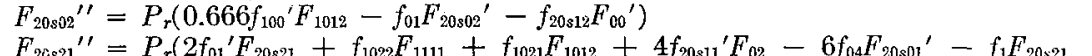 & $F_{20 \leqslant 02}$ & $=0$ & $F_{20, s 02}$ & $=$ \\
\hline & $\begin{array}{r}\left.-2 f_{1022} F_{1011}^{\prime}-2 f_{20 s 11} F_{02}^{\prime}-2 f_{1021} F_{1012}+2 f_{100} F_{1212}-3 f_{20 s 33} F_{00}{ }^{\prime}\right)\end{array}$ & $F_{20821}$ & $=0$ & $F_{20 s 21}$ & $=$ \\
\hline$F_{20 s 22}$ & $F_{20 s 22}{ }^{\prime \prime}=P_{r}\left(2 f_{01}{ }^{\prime} F_{\mathrm{g} 0 s 22}+f_{1021}{ }^{\prime} F_{1011}-f_{01} F_{20 s 22}{ }^{\prime}-2 f_{1021} F_{1011}^{\prime}-3 f_{20 s 22} F_{00}{ }^{\prime}\right)$ & $F_{20 s 22}$ & $=0$ & $F_{20 s 22}$ & $=$ \\
\hline$F_{20 s 23}$ & $\begin{array}{c}F_{20 s 23}{ }^{\prime \prime}=P_{r}\left(2 f_{01}{ }^{\prime} F_{20 s 23}+f_{1022} F_{1012}+4 f_{20 s 12}{ }^{\prime} F_{02}-6 f_{04} F_{20 s 02}-f_{01} F_{20 s 23}{ }^{\prime}-2 f_{1022} F_{1012}{ }^{\prime}\right. \\
\left.-2 f_{20 s 12} F_{02}^{\prime}+2 f_{100} F_{1034}-3 f_{20 s 34} F_{00}{ }^{\prime}\right)\end{array}$ & $F_{20 s 23}$ & $=0$ & $F_{20 s 23}$ & $=$ \\
\hline$F_{20824}$ & $F_{20 s 24}{ }^{\prime \prime}=P_{r}\left(2 f_{01}{ }^{\prime} F_{20 s 24}-f_{01} F_{20 s 24}^{\prime}+2 f_{100}{ }^{\prime} F_{1021}-3 f_{20 s 31} F_{00}{ }^{\prime}\right)$ & $F_{20 s 24}$ & $=0$ & $F_{201824}$ & $=$ \\
\hline$F_{20325}$ & $F_{20 s 25}^{\prime \prime}=P_{r}\left(2 f_{01}^{\prime} F_{20 s 25}-f_{01} F_{20 s 25}^{\prime}+2 f_{100}^{\prime} F_{1033}-3 f_{s 0235} F_{00}^{\prime}\right)$ & $F_{20 s 25}$ & $=0$ & $F_{2 t s 25}$ & $=$ \\
\hline
\end{tabular}

$$
\begin{gathered}
\frac{\partial u_{2}}{\partial x}+\frac{\partial v_{2}}{\partial y}=0 \\
\frac{\partial u_{2}}{\partial t}+u_{2} \frac{\partial u_{0}}{\partial x}+u_{1} \frac{\partial u_{1}}{\partial x}+u_{0} \frac{\partial u_{2}}{\partial x}+v_{2} \frac{\partial u_{0}}{\partial y}+v_{1} \frac{\partial u_{1}}{\partial y}+ \\
v_{0} \frac{\partial u_{2}}{\partial y}=\frac{U_{1}}{2} \frac{d U_{1}}{d x}(1+\cos 2 \omega t)+\frac{1}{R e} \frac{\partial^{2} u_{2}}{\partial y^{2}} \\
\frac{\partial T_{2}}{\partial t}+u_{0} \frac{\partial T_{2}}{\partial x}+u_{1} \frac{\partial T_{1}}{\partial x}+u_{2} \frac{\partial T_{0}}{\partial x}+v_{0} \frac{\partial T_{2}}{\partial y}+ \\
v_{1} \frac{\partial T_{1}}{\partial y}+v_{2} \frac{\partial T_{0}}{\partial y}=\frac{1}{\operatorname{RePr}} \frac{\partial^{2} T_{2}}{\partial y^{2}} \\
y=u_{2}=v_{2}=T_{2}=0 \\
y=\infty: u_{2}=T_{2}=0
\end{gathered}
$$

Table 2 Universal functions $\boldsymbol{f}$ and $\boldsymbol{F}$ for quasi-steady solutions 
Here the value of $\epsilon$ is chosen small such that the first three terms of the expansion will approximate the physical problem.

\section{A. Zeroth-Order Solutions}

The zeroth-order perturbation is the case of steady-state forced convection. ${ }^{20}$ In two-dimensional flow, the timeaverage freestream velocity follows the formula

$$
U_{0}(x)=\sum_{k=0}^{\infty} a_{2 k+1} x^{2 k+1}
$$

where the coefficients $a_{2 k+1}$, which depend on the geometrical configuration of the body, are considered to be known. By selecting the dimensionless distance from the wall, defined as $\eta=y\left(a_{1} R e\right)^{1 / 2}$ as the similarity variable, the stream function and temperature are obtained:

$$
\left.\begin{array}{l}
\psi_{0}=\left(\frac{a_{1}}{R e}\right)^{1 / 2}\left(f_{01} x+\frac{4 a_{3}}{a_{1}} f_{03} x^{3}+\frac{6 a_{5}}{a_{1}} f_{05} x^{5}+\ldots\right) \\
T_{0}=\theta_{0}=F_{00}+\frac{4 a_{3}}{a_{1}} F_{02} x^{2}+\frac{6 a_{5}}{a_{1}} F_{04} x^{4}+\ldots
\end{array}\right\}
$$

The subscripts for the universal functions $f$ and $F$ are described in Table 1. Equations and their appropriate boundary conditions that define the functions $f$ and $F$ in Eq. (7) and later in Eqs. (11) and (14) are given in Ref. 21. Only those relevant to the quasi-steady solutions are tabulated in Table 2.

\section{B. First-Order Solutions}

For low frequencies, the velocity and temperature components are expanded in terms of frequency as follows. It is convenient to adopt complex notations and to write

$$
\left.\begin{array}{rl}
u_{1}(x, y, t)=\operatorname{real}\left\{\left[u_{10}(x, y)+\right.\right. & (i \omega) u_{11}(x, y)+ \\
& \left.\left.(i \omega)^{2} u_{12}(x, y)+\ldots\right] e^{i \omega t}\right\} \\
v_{1}(x, y, t)=\operatorname{real}\left\{\left[v_{10}(x, y)+\right.\right. & (i \omega) v_{11}(x, y)+ \\
& \left.\left.(i \omega)^{2} v_{12}(x, y)+\ldots\right] e^{i \omega t}\right\} \\
T_{1}(x, y, t)=\operatorname{real}\left\{\left[\theta_{10}(x, y)+\right.\right. & (i \omega) \theta_{11}(x, y)+ \\
& \left.\left.(i \omega)^{2} \theta_{12}(x, y)+\ldots\right] e^{i \omega t}\right\}
\end{array}\right\}
$$

The new funetions $u_{10}, v_{10}, \theta_{10}, \ldots$ are now complex quantities and independent of time. Substituting Eq. (8) into Eq. (4) and observing that the symbol "real" appears in front of every term, it is disclosed that, since "real" is a linear operator, it can be dropped out of the equations. All terms are now linear in $e^{i \omega t}$, which can also be dropped out. In this manner, timedependency is omitted from the differential equations. Arranging the time-independent equations according to the powers of $i \omega$ results in the $l$ th-order approximation $(i \omega)^{l}$ :

$$
\begin{gathered}
\frac{\partial u_{1 l}}{\partial x}+\frac{\partial v_{1 l}}{\partial y}=0 \\
u_{0} \frac{\partial u_{1 l}}{\partial x}+u_{1 l} \frac{\partial u_{0}}{\partial x}+v_{0} \frac{\partial u_{1 l}}{\partial y}+v_{1 l} \frac{\partial u_{0}}{\partial y}= \\
\frac{1}{R e} \frac{\partial^{2} u_{1 l}}{\partial y^{2}}+ \begin{cases}U_{0} \frac{\partial U_{1}}{d x}+U_{1} \frac{d U_{0}}{d x} & \text { for } l=0 \\
U_{1}-u_{10} & \text { for } l=1 \\
-u_{1(l-1)} & \text { for } l>1 \\
u_{0} \frac{\partial \theta_{1 l}}{\partial x}+u_{1 l} \frac{\partial \theta_{0}}{\partial x}+v_{0} \frac{\partial \theta_{1 l}}{\partial y}+v_{1 l} \frac{\partial \theta_{0}}{\partial y}= & \text { for } l=0 \\
\frac{1}{R e P r} \frac{\partial^{2} \theta_{1 l}}{\partial y^{2}}+ \begin{cases}0 & \text { for } l>0 \\
\theta_{1(l-1)} & l\end{cases} \\
y=0: u_{1 l}=v_{1 l}=\theta_{1 l}=0\end{cases} \\
y=\infty: u_{10}=U_{1}, u_{1 l}=\theta_{1 l}=0
\end{gathered}
$$

where $l$ is the order of approximation $(i \omega)$. The amplitude follows the formula

$$
U_{1}(x)=\sum_{k=0}^{\infty} b_{2 k} x^{2 k}
$$

where the coefficients $b_{2 k}$ depend upon the nature of the fluctuating circulations. $U_{1}(x)$ is an even-power series, since only the velocity fluctuations of the potential flow caused by a fluctuating circulation are considered. Then the solutions of Eq. (4) are obtained as

$$
\left.\begin{array}{l}
\psi_{1 l}=\frac{1}{a_{1}^{l}\left(a_{1} R e\right)^{1 / 2}}\left(b_{0} f_{1 l l}+3 b_{22} f_{1 l 2} x^{2}+5 b_{4} f_{1 l 4} x^{4}+\ldots\right) \\
\theta_{1 l}=\frac{2}{a_{1}^{l+1}}\left(b_{2} F_{1 l 1} x+2 b_{4} F_{133} x^{3}+4 b_{6} F_{1 l 5} x^{5}+\ldots\right)
\end{array}\right\}
$$

where $l$ is the order of approximation $(i \omega)$. The functional coefficients $f_{1 l k}$ and $F_{1 l k}$ are independent of the particular properties of the profiles (i.e., $a$ ), and of the fluctuating circulation (i.e., $b$ ), if they are split up as

$$
\begin{gathered}
f_{1 l 2}=f_{1 l 21}+\left(a_{3} b_{0} / a_{1} b_{2}\right) f_{1 l 22} \\
f_{1 l 4}=f_{1 l 41}+\frac{a_{3} b_{2}}{a_{1} b_{4}} f_{1 l 32}+\frac{a_{5} b_{0}}{a_{1} b_{4}} f_{1 l 43}+\frac{b_{0} a_{3}^{2}}{a_{1}^{2} b_{4}} f_{1 l 44}
\end{gathered}
$$

and

$$
\begin{gathered}
F_{1 l 1}=F_{1111}+\left(a_{3} b_{0} / a_{1} / b_{2}\right) F_{1 l 12} \\
F_{1 l 3}=F_{1231}+\frac{a_{3} b_{2}}{a_{1} b_{4}} F_{1 l 32}+\frac{a_{5} b_{0}}{a_{1} b_{4}} F_{1233}+\frac{b_{0} a_{3}^{2}}{a_{1}^{2} b_{4}} F_{1 l 34}
\end{gathered}
$$

\section{Second-Order Solutions}

In the second-order perturbation for $u_{2}(x, y, t), v_{2}(x, y, t)$, and $T_{2}(x, y, t)$, the convective terms in the governing equations will contribute terms with $\cos ^{2} \omega t$. These, in turn, can be reduced to terms with $\cos 2 w t, \sin 2 w t$, and steady-state, i.e., time-independent terms. ${ }^{18}$ Therefore $u_{2}, v_{2}$, and $T_{2}$, for low frequencies, may be expanded as follows:

$$
\left.\begin{array}{rl}
u_{2}(x, y, t)= & \frac{1}{2} \operatorname{real}\left\{u_{20 s}(x, y)+(i \omega) u_{21 s}(x, y)+\right. \\
(i \omega)^{2} u_{2 s 2}(x, y)+\ldots+\left[u_{20 t}(x, y)+\right. \\
\left.\left.(i \omega) u_{21 t}(x, y)+(i \omega)^{2} u_{22 t}(x, y)+\ldots\right] e^{i 2 \omega t}\right\} \\
v_{2}(x, y, t)=\frac{1}{2} \operatorname{real}\left\{v_{20 s}(x, y)+(i \omega) v_{21 s}(x, y)+\right. \\
(i \omega)^{2} v_{22 s}(x, y)+\ldots\left[v_{20 t}(x, y)+\right. \\
\left.\left.(i \omega) v_{21 t}(x, y)+(i \omega)^{2} v_{22 t}(x, y)+\ldots\right] e^{i 2 \omega t}\right\} \\
\theta_{2}(x, y, t)=\frac{1}{2} \operatorname{real}\left\{\theta_{20 s}(x, y)+(i \omega) \theta_{21 s}(x, y)+\right. \\
(i \omega)^{2} \theta_{22 s}(x, y)+\ldots+\left[\theta_{20 t}(x, y)+\right. \\
\left.\left.(i \omega) \theta_{21 t}(x, y)+(i \omega)^{2} \theta_{22 t}+\ldots\right] e^{i 2 \omega t}\right\}
\end{array}\right\}
$$

The equations of continuity, momentum, and energy, which are obtained by substituting these relationships into Eq. (5) and by separating according to frequency- and time-dependency, are as follows. Although the analysis has been extended to include the first- and second-order approximations, only two resulting time-dependent and time-independent equations corresponding to the zeroth-order approximation $(i \omega)^{0}$ are presented here. They are

$$
\begin{gathered}
\frac{\partial u_{20 j}}{\partial x}+\frac{\partial v_{20 j}}{\partial y}=0 \\
u_{0} \frac{\partial u_{20 i}}{\partial x}+u_{10} \frac{\partial u_{10}}{\partial x}+u_{20 j} \frac{\partial u_{0}}{\partial x}+v_{0} \frac{\partial u_{20 j}}{\partial y}+ \\
v_{10} \frac{\partial u_{10}}{\partial y}+v_{20 j} \frac{\partial u_{0}}{\partial y}=\frac{1}{R e} \frac{\partial^{2} u_{20 j}}{\partial y^{2}}+U_{1} \frac{d U_{1}}{d x}
\end{gathered}
$$

(Equation continued on following page) 


$$
\begin{gathered}
u_{0} \frac{\partial \theta_{20 j}}{\partial x}+u_{10} \frac{\partial \theta_{10}}{\partial x}+u_{20 j} \frac{\partial \theta_{0}}{\partial x}+v_{0} \frac{\partial \theta_{20 i}}{\partial y}+ \\
v_{10} \frac{\partial \theta_{10}}{\partial y}+v_{20 j} \frac{\partial \theta_{0}}{\partial y}=\frac{1}{\operatorname{RePr}} \frac{\partial^{2} \theta_{20 i}}{\partial y^{2}} \\
y=0: u_{20 j}=v_{20 j}=\theta_{20 j}=0 \\
y=\infty: u_{20 j}=\theta_{20 j}=0
\end{gathered}
$$

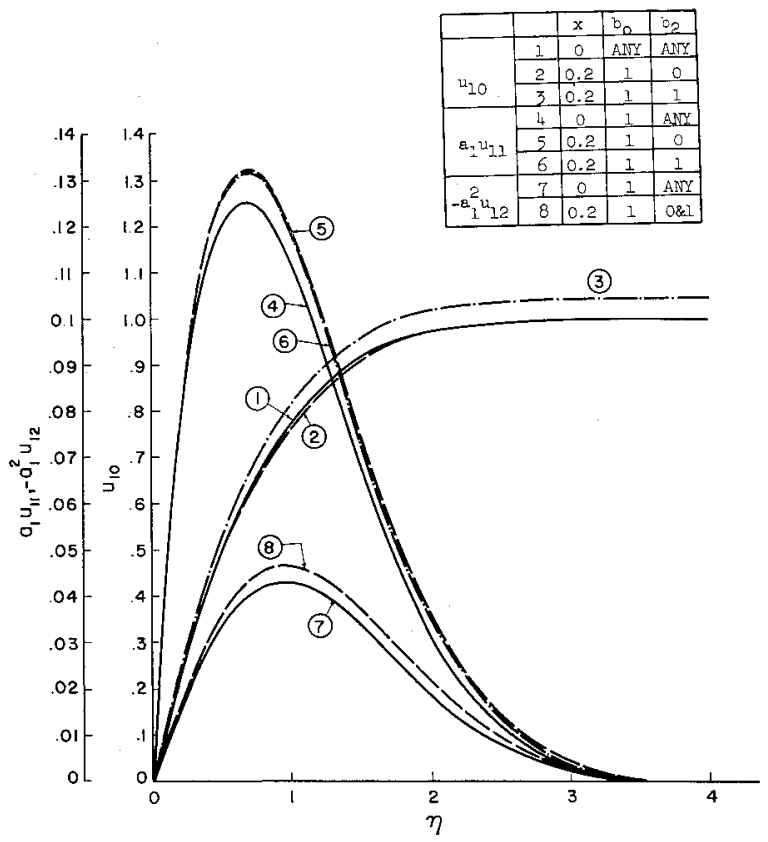

Fig. la Profiles of first-order velocities for flow past a circular cylinder with fluctuating circulations.

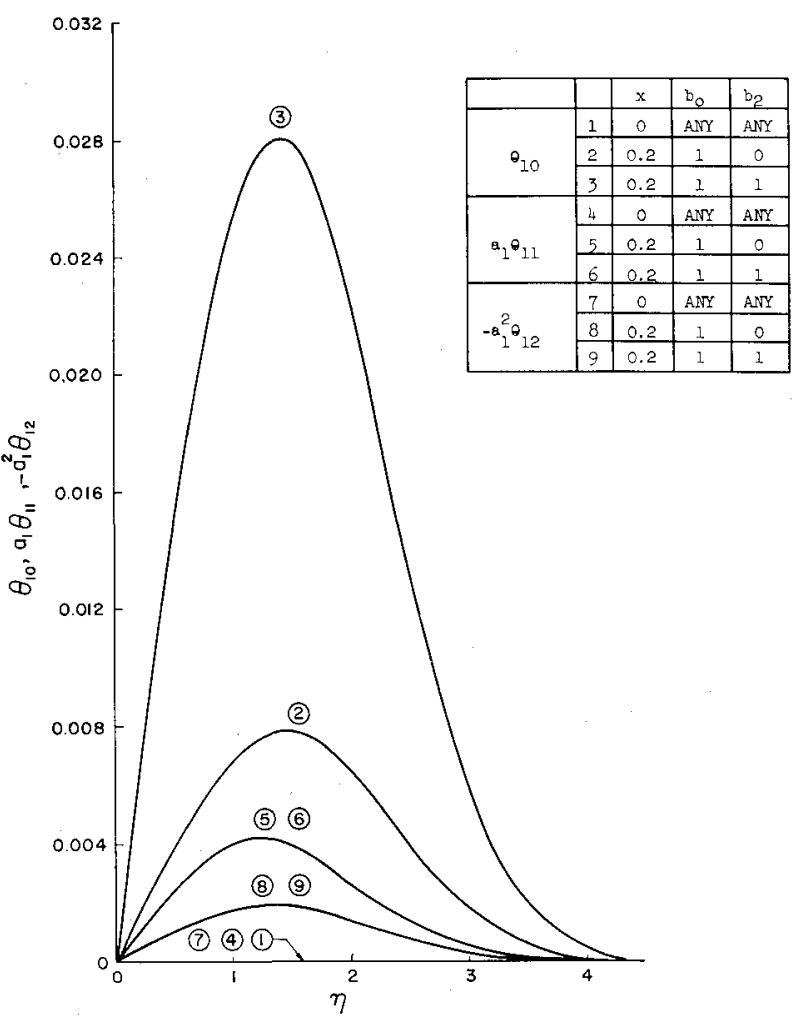

Fig. 1b Profiles of first-order temperatures or concentrations for flow past a circular cylinder with fluctuating circulations for a fluid with $P r=1.0$ or $S c=1.0$. where the subscript $j$ refers to either the time-independent or time-dependent components, respectively expressed by the subscripts $s$ and $t$.

The second-order solutions are as follows:

$$
\begin{aligned}
& \psi_{21 j}=\frac{3 b_{0} b_{2}}{a_{1}{ }^{l+1}\left(a_{1} R e\right)^{1 / 2}}\left(f_{2 l i 1} x+\frac{2 a_{3}}{a_{1}} f_{2 l j 3} x^{3}+\right. \\
& \left.\frac{3 a_{5}}{a_{1}} f_{2 l j 5} x_{5}+\ldots\right) \\
& \theta_{2 l j}=\frac{3 b_{0} b_{2}}{a_{1}{ }^{l+2}}\left(F_{2 l j 0}+\frac{2 a_{3}}{a_{1}} F_{2 l j 2} x^{2}+\right. \\
& \left.\frac{3 a_{5}}{a_{1}} F_{2 l j 4} x^{4}+\ldots\right)
\end{aligned}
$$

where

$$
f_{2 l i 1}=f_{2 l i 11}+\left(a_{3} b_{0} / a_{1} b_{2}\right) f_{2 l j 12}
$$

$f_{2 l j 3}=f_{2 l j 31}+\frac{a_{3} b_{0}}{a_{1} b_{2}} f_{2 l j 32}+\frac{a_{1} b_{2}}{a_{3} b_{0}} f_{2 l j 33}+\frac{a_{1} b_{4}}{a_{3} b_{2}} f_{2 l j 34}+\frac{a_{5} b_{0}}{a_{3} b_{2}} f_{2 l j 35}$ and

$$
\begin{gathered}
F_{2 l j 0}=F_{2 l j 01}+\left(a_{3} b_{0 /} a_{1} b_{2}\right) F_{2 l j 02} \\
F_{2 l j 2}=F_{2 l j 21}+\frac{a_{3} b_{0}}{a_{1} b_{2}} F_{2 l j 22}+\frac{a_{1} b_{2}}{a_{3} b_{0}} F_{2 l j 23}+ \\
\frac{a_{1} b_{4}}{a_{3} b_{2}} F_{2 l j 24}+\frac{a_{5} b_{0}}{a_{3} b_{2}} F_{2 l j 25}
\end{gathered}
$$

\section{Solutions Up to Second Order}

The velocity components are

$$
\begin{gathered}
\left.\begin{array}{c}
u=u_{0}+\epsilon\left[\left(u_{10}-\omega^{2} u_{12}\right)^{2}+\left(\omega u_{11}\right)^{2}\right]^{1 / 2} \times \\
\cos \left[\omega t+\tan ^{-1}\left(\frac{\omega u_{11}}{u_{10}-\omega^{2} u_{12}}\right)\right]+ \\
\frac{\epsilon^{2}}{2}\left\{u_{20 s}-\omega^{2} u_{22 s}+\left[\left(u_{20 t}-\omega^{2} u_{22 t}\right)^{2}+\left(\omega u_{21 t}\right)^{2}\right]^{1 / 2} \times\right. \\
\left.\cos \left[2 \omega t+\tan ^{-1}\left(\frac{\omega u_{21 t}}{u_{20 t}-\omega^{2} u_{22 t}}\right)\right]\right\} \\
v=v_{0}+\epsilon\left[\left(v_{10}-\omega^{2} v_{12}\right)^{2}+\left(\omega v_{11}\right)^{2}\right]^{1 / 2} \times \\
\cos \left[\omega t+\tan ^{-1}\left(\frac{\omega v_{11}}{v_{10}-\omega^{2} v_{12}}\right)\right]+ \\
\frac{\epsilon^{2}}{2}\left\{v_{20 s}-\omega^{2} v_{22 s}+\left[\left(v_{20 t}-\omega^{2} v_{22 t}\right)^{2}+\left(\omega v_{21 t}\right)^{2}\right]^{1 / 2} \times\right. \\
\left.\cos \left[2 \omega t+\tan ^{-1}\left(\frac{\omega v_{21 t}}{v_{20 t}-\omega^{2} v_{22 t}}\right)\right]\right\}
\end{array}\right\}
\end{gathered}
$$

The dimensionless shear stress, defined as $\tau=(\partial u / \partial y)_{y=0}$, is

$$
\begin{gathered}
\tau=\left\{\frac{\partial u_{0}}{\partial y}+\epsilon\left[\left(\frac{\partial u_{10}}{\partial y}-\omega^{2} \frac{\partial u_{12}}{\partial y}\right)^{2}+\right.\right. \\
\left.\left(\omega \frac{\partial u_{11}}{\partial y}\right)^{2}\right]^{1 / 2} \cos \left[\omega t+\tan ^{-1}\left(\frac{\omega\left(\partial u_{11} / \partial y\right)}{\left(\partial u_{10} / \partial y\right)-\omega^{2}\left(\partial u_{12} / \partial y\right)}\right)+\right. \\
\frac{\epsilon^{2}}{2}\left(\frac{\partial u_{20 s}}{\partial y}-\omega^{2} \frac{\partial u_{22 s}}{\partial y}\right)+\frac{\epsilon^{2}}{2}\left(\frac{\partial u_{20 t}}{\partial y}-\omega^{2} \frac{\partial u_{22 t}}{\partial y}\right)^{2}+ \\
\left.\left(\omega \frac{\partial u_{21 t}}{\partial y}\right)\right]^{1 / 2} \cos [2 \omega t+ \\
\left.\left.\tan ^{-1}\left(\frac{\omega\left(\partial u_{21} / \partial y\right)}{\left(\partial u_{20} / \partial y\right)-\left(\partial u_{22} / \partial y\right) \omega^{2}}\right)\right]\right\}_{y=0}
\end{gathered}
$$

The steady-state shear stress is Eq. (16) without the second and fourth terms on the right-hand side of the equation. The periodic terms will contribute nothing when integrated over a whole cycle. The mean position of separation may be ob- 
tained by equating the steady-state shear stress to zero. The separation in the unsteady boundary layer is fully discussed by Hori. ${ }^{3}$ The temperature is

$$
\begin{gathered}
T=\theta_{0}+\epsilon\left[\left(\theta_{10}-\omega^{2} \theta_{12}\right)^{2}+\left(\omega \theta_{11}\right)^{2}\right]^{1 / 2} \times \\
\cos \left[\omega t+\tan ^{-1}\left(\frac{\omega \theta_{11}}{\theta_{10}-\omega^{2} \theta_{12}}\right)\right]+ \\
\frac{\epsilon^{2}}{2}\left\{\theta_{20 s}-\omega^{2} \theta_{22 s}+\left[\left(\theta_{20 t}-\omega^{2} \theta_{22 t}\right)^{2}+\left(\omega \theta_{21 t}\right)^{2}\right]^{1 / 2} \times\right. \\
\left.\cos \left[2 \omega t+\tan ^{-1}\left(\frac{\omega \theta_{21 t}}{\theta_{20 t}-\omega^{2} \theta_{22 t}}\right)\right]\right\}
\end{gathered}
$$

The local Nusselt number, defined as $N u=(\partial T / \partial v)_{y=0}$, is obtained as

$$
\begin{array}{r}
N_{u}=\left\{\frac{\partial \theta_{0}}{\partial y}+\epsilon\left[\left(\frac{\partial \theta_{0}}{\partial y}-\omega^{2} \frac{\partial \theta_{12}}{\partial y}\right)^{2}+\left(\omega \frac{\partial \theta_{11}}{\partial y}\right)^{2}\right]^{1 / 2} \times\right. \\
\cos \left[\omega t+\tan ^{-1}\left(\frac{\omega\left(\partial \theta_{11} / \partial y\right)}{\left(\partial \theta_{10} / \partial y\right)-\omega^{2}\left(\partial \theta_{12} / \partial y\right)}\right)\right]+ \\
\frac{\epsilon^{2}}{2}\left(\frac{\partial \theta_{20 s}}{\partial y}-\omega^{2} \frac{\partial \theta_{22 s}}{\partial y}\right)+\frac{\epsilon^{2}}{2}\left[\left(\frac{\partial \theta_{20 t}}{\partial y}-\omega^{2} \frac{\partial \theta_{22 t}}{\partial y}\right)^{2}+\right. \\
\left.\left(\omega \frac{\partial \theta_{21 t}}{\partial y}\right)^{2}\right]^{1 / 2} \cos [2 \omega t+ \\
\left.\left.\tan ^{-1}\left(\frac{\omega\left(\partial \theta_{21 t} / \partial y\right)}{\left(\partial \theta_{20 t} / \partial y\right)-\omega^{2}\left(\partial \theta_{22 t} / \partial y\right)}\right)\right]\right\}_{y=0}
\end{array}
$$

Again the periodic terms contribute nothing to the steadystate value.

\section{E. Net Alterations in Skin-Friction and Heat-Transfer Rate Resulting from Fluctuating Circulations}

Equations (15) indicate that a fluctuating circulation induces a steady-secondary or streaming motion; Eq. (18) shows that the streaming flow resulting from the oscillation causes a change in the local Nusselt number from that corresponding to steady forced convection. The second-order contributions to the net variations of the wall shear stress and heat-transfer rate are obtained from Eqs. $(14,16$, and 18) as follows:

$$
\begin{aligned}
& \frac{\Delta \tau}{(R e)^{1 / 2}}=\frac{3 \epsilon^{2} b_{0} b_{2}}{2 a_{1}^{1 / 2}} x\left\{f_{20 s 11}{ }^{\prime \prime}+\frac{b_{0} a_{3}}{a_{1} b_{2}} f_{20 s 12}^{\prime \prime}-\right. \\
& \left(\frac{\omega}{a_{1}}\right)^{2}\left(f_{22 s 11}^{\prime \prime}+\frac{a_{3} b_{0}}{a_{1} b_{2}} f_{22 s 12}^{\prime \prime}\right)+ \\
& \frac{2 a_{3}}{a_{1}}\left[f_{20831}{ }^{\prime \prime}+\frac{a_{3} b_{0}}{a_{1} b_{2}} f_{2083 z} z^{\prime \prime}+\frac{a_{1} b_{2}}{a_{3} b_{0}} f_{20833}{ }^{\prime \prime}+\frac{a_{1} b_{4}}{a_{3} b_{2}} f_{20834}{ }^{\prime \prime}+\right. \\
& \frac{a_{5} b_{0}}{a_{3} b_{2}} f_{20 s 85}{ }^{\prime \prime}-\left(\frac{\omega}{a_{1}}\right)^{2}\left(f_{22 s 31}{ }^{\prime \prime}+\frac{a_{3} b_{0}}{a_{1} b_{2}} f_{22 s 32}{ }^{\prime \prime}+\right. \\
& \left.\left.\left.\frac{a_{1} b_{2}}{a_{3} b_{0}} f_{22833}{ }^{\prime \prime}+\frac{a_{1} b_{4}}{a_{3} b_{2}} f_{22534}{ }^{\prime \prime}+\frac{a_{5} b_{0}}{a_{3} b_{2}} f_{22 s 35}\right)\right] x^{2}+\ldots\right\}_{\eta=0} \\
& \frac{\Delta N_{u}}{(R e)^{1 / 2}}=\frac{3 \epsilon^{2} b_{0} b_{2}}{2 a_{1}^{2}}\left\{F_{20 s 01}^{\prime}+\frac{a_{3} b_{0}}{a_{1} b_{2}} F_{20 s 02}{ }^{\prime}-\right. \\
& \frac{2}{3}\left(\frac{\omega}{a_{1}}\right)^{2}\left(F_{22} s_{01}^{\prime}+\frac{a_{3} b_{0}}{a_{1} b_{2}} F_{22 s 02}^{\prime}\right)+ \\
& \frac{2 a_{3}}{a_{1}}\left[F_{20 s 21}{ }^{\prime}+\frac{a_{1} b_{2}}{a_{3} b_{0}} F_{20 s 23}^{\prime}+\frac{a_{3} b_{0}}{a_{1} b_{2}} F_{20 s 22}{ }^{\prime}+\right. \\
& \frac{a_{1} b_{4}}{a_{3} b_{2}} F_{20 s 24}^{\prime}+\frac{a_{5} b_{0}}{a_{3} b_{2}} F_{20 s 25}^{\prime}-\left(\frac{\omega}{a_{1}}\right)^{2}\left(F_{22 \Omega 21}^{\prime \prime}+\frac{a_{1} b_{2}}{a_{3} b_{0}} F_{22 s 23}{ }^{\prime}+\right. \\
& \left.\left.\left.\frac{a_{3} b_{0}}{a_{1} b_{2}} F_{22 s 22^{\prime}}+\frac{a_{1} b_{4}}{a_{3} b_{2}} F_{22 s 24}^{\prime}+\frac{a_{5} b_{0}}{a_{3} b_{2}} F_{20 s 25^{\prime}}^{\prime}\right)\right] x^{2}+\ldots\right\}_{\eta=0}
\end{aligned}
$$

\section{Results and Discussion}

Table 2 presents the differential equations and their appropriate boundary conditions which define the universal functions $f$ and $F$. In the interest of brevity, only those functions relevant to the quasi-steady solutions, i.e, $\omega \rightarrow 0$, are given. As will be discussed later in the section, these quasi-steady solutions may approximate the behavior of the unsteady boundary layers. All functions $f$ and $F$ in Eqs. $(7,11$, and 14) were evaluated by steps of $\eta=0.1$ using an IBM 7090 digital computer. The method of Runge and Kutta was employed for this purpose. Their numerical reduction was obtained for Prandtl numbers from 1.0 to 10 and $V=0,1,-1$. A positive $V$ corresponds to the superposition of uniform blowing, and a negative $V$ corresponds to that of uniform suction.

Owing to the dependency of transfer performance on the coefficients $a$ and $b$, the geometrical configuration of the system and the nature of fluctuating circulation must be specified for the quantitative as well as qualitative evaluation of the mechanics of two-dimensional flow. As an illustration, the case of a uniform flow about an infinite circular cylinder with a superposition of an alternating vortex placed in the stream is treated.

The time-average mean velocity of the external potential flow for this case is

$$
U_{0}(x)=2 \sin (2 x)
$$

By expanding the sine function on the right-hand side of the equation into a power series, one obtains the coefficients $a_{2 k+1}$ as

$$
a_{1}=4, a_{3}=-\frac{8}{3}, a_{5}=\frac{8}{15}, \ldots
$$

Next a model must be selected for the alternating vortex in order to determine the coefficients $b_{2 k}$. In an experimental study of the general characteristics of fluctuating pressures (caused by shedding vortices) at the surface of a circular cylinder in a uniform stream, McGregor ${ }^{22}$ has developed a simple mathematical model of the flow by considering an alternating vortex standing at the rear of the cylinder. The theoretical prediction of the pressure fluctuations based on this model was in good agreement with the experimental results; however, it requires at least a fourth-degree polynomial to approximate the model in the form of $U_{1}(x)$. In a study of unsteady flow in boundary layers, Hori ${ }^{3}$ has analyzed a special case of the McGregor's model in which the vortex in the stream is shifted to infinity. For this case, the amplitude of fluctuating circulations becomes a constant. In the present work, two different oscillating amplitudes $\epsilon U_{1}(x)$ are investigated: 1) constant and equal to $\epsilon$, for which $b_{0}=1, b_{2}=b_{4}=$ $\ldots=0 ; 2)$ space-dependent and equal to either $\epsilon\left(1+x^{2}\right)$, for which $b_{0}=b_{2}=1, b_{4}=b_{6}=\ldots=0$, or $\epsilon\left(1+x^{2}+x^{4}\right)$, for which $b_{0}=b_{2}=b_{4}=1, b_{6}=b_{8}=\ldots=0$. It must be noted that $U_{1}(x)$ in the latter case does not mathematically approximate McGregor's model. Nevertheless, it is adopted in order to numerically demonstrate the effect of the spacedependency of the fluctuating amplitude on the transfer phenomena.

The stream functions and temperatures defined by Eqs. $(7,11$, and 14) are calculated with three terms for the zerothand first-order solutions and two terms for the second-order solution. This limitation on the number of terms involved is mainly attributed to the computer capacity. Since there is no general method to determine the radius of convergence, the quality of the power-series solution has to be evaluated by studying the behavior of the series with various number of terms. ${ }^{23}$ An examination disclosed that, after the first term, each series converges well, especially near the surface for small values of $x$. For large values of $x$, all series give fairly good convergence with three terms except in the neighborhood of the separation point. In general, the stream functions give better convergence than the temperature solutions with the 
same number of terms. The convergence of Eqs. (19) for the net alterations in skin friction and heat-transfer rate is quite good with the first two $x$ terms, especially for low values of $x$.

Numerical results are presented in graphical form in Figs. 1-5. The zeroth-order solutions are exactly those of Fröss$\operatorname{lin}^{20}$ for the steady-state forced convection. The first-order

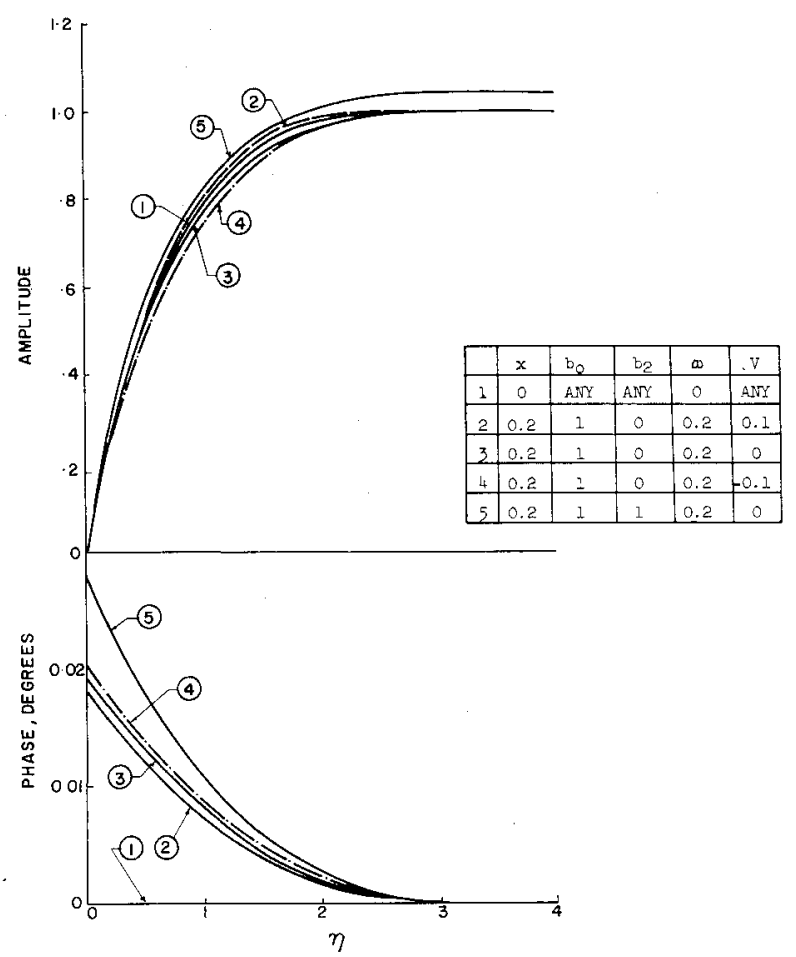

Fig. 2a Amplitude and phase or fluid velocity for flow past a circular cylinder with fluctuating circulations.

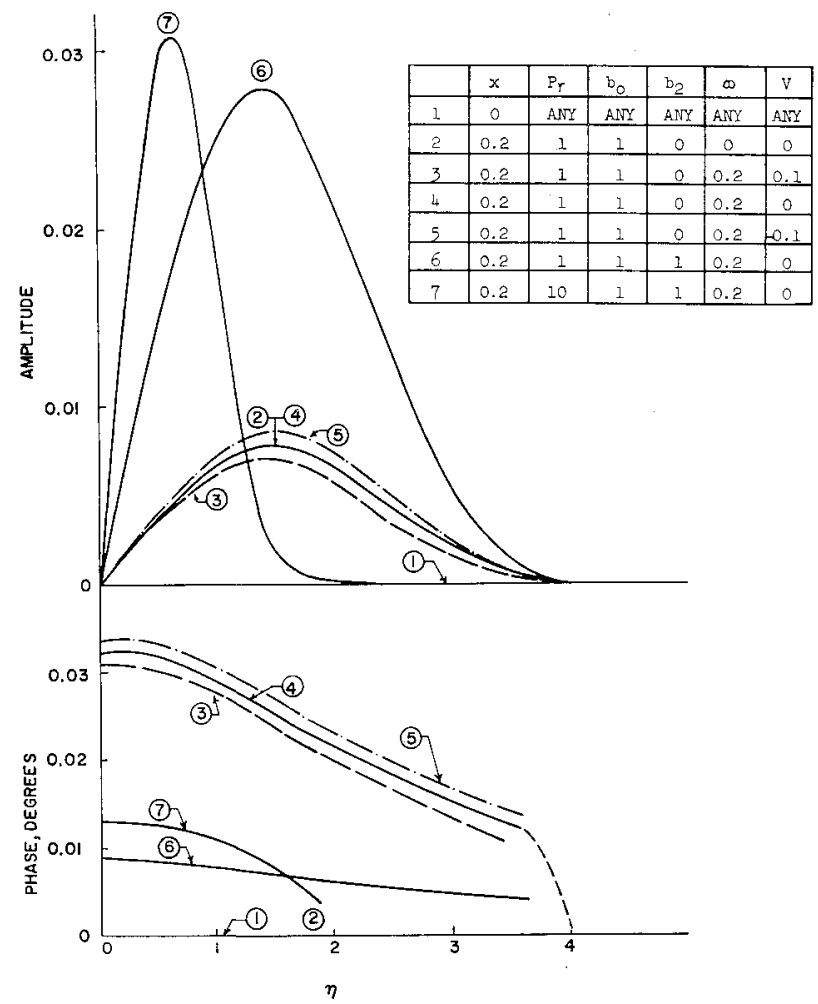

Fig. 2b Amplitude and phase of fluid temperature or concentration for flow past a circular cylinder with fluctuating circulations. solutions give the magnitude and phase angle of harmonic oscillations of velocity and temperature inside the boundary layer induced by the fluctuating circulations. Figure 1 gives the first-order velocity and temperature profiles $u_{1 l}$ and $\theta_{1 l}$. $u_{10}$ (and $v_{10}$ ) and $\theta_{10}$ are the quasi-steady velocity and temperature, respectively, that is, the amplitudes of velocity and temperature fluctuations at $\omega \rightarrow 0 . i \omega u_{11},(i \omega)^{2} u_{12}$ [and also $\left.i \omega v_{11},(i \omega)^{2} v_{12}\right]$, and $(i \omega) \theta_{11},(i \omega)^{2} \theta_{12}$ represent the amplitudes of unsteady velocity and temperature fluctuations, respectively. It is disclosed by Figs. 1 that both the velocity profiles $u_{10}, u_{11}$, $u_{12}$ and the temperature profiles $\theta_{10}, \theta_{11}, \theta_{12}$ increase in magnitude along the surface from the forward stagnation point. Since $U_{1}=b_{0}$ corresponds to the case of the alternating vortex located at infinity, $U_{1}=b_{0}+b_{2} x^{2}$ or $b_{0}+b_{2} x^{2}+b_{4} x^{4}$ represents the case in which this vortex has been shifted toward the cylinder. The shift in the vortex from infinity toward the cylinder results in an increase in the quasi-steady amplitudes of both velocity and temperature, but practically no significant variations in the amplitudes of unsteady velocity and temperature fluctuations.

Figures $2 \mathrm{a}$ and $2 \mathrm{~b}$ illustrate the amplitude and phase angle of the first-order harmonic oscillations of velocity $u_{1}$ and temperature $\theta_{1}$. The effects of several physical factors on the frequency response of velocity and temperature are investigated. They include the location $x$ measured from the forward stagnation point, the location of the vortex, the uniform blowing or suction of the boundary layer, and the Prandtl number. It is seen from Fig. 2 that in general the amplitude and phase angle of both velocity and temperature fluctuations increase with increases in $x$, frequency, and the shift in the

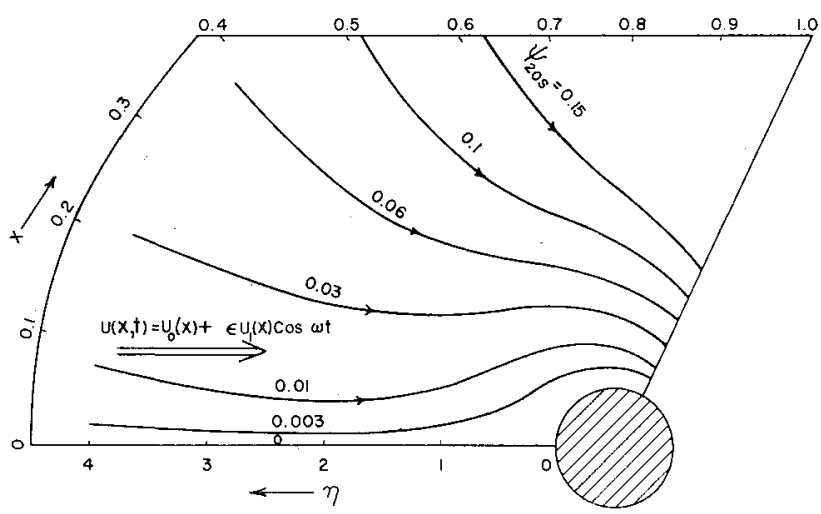

Fig. 3a Streamline pattern of the steady-secondary motion produced by flow past a circular cylinder with fluctuating circulations of amplitude, $\epsilon \boldsymbol{u}_{1}=\epsilon$, at quasi-steady state $(\omega \rightarrow 0)$.

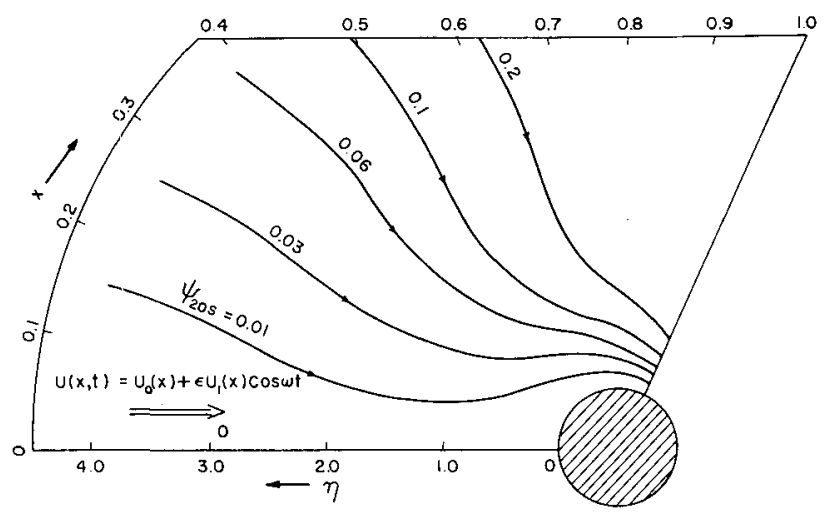

Fig. 3b Streamline pattern of the steady-secondary motion produced by flow past a circular cylinder with fluctuating circulation of amplitude, $u_{1}(x)=\epsilon\left(1+x+x^{2}\right)$, at quasi-steady state $(\omega \rightarrow 0)$. 


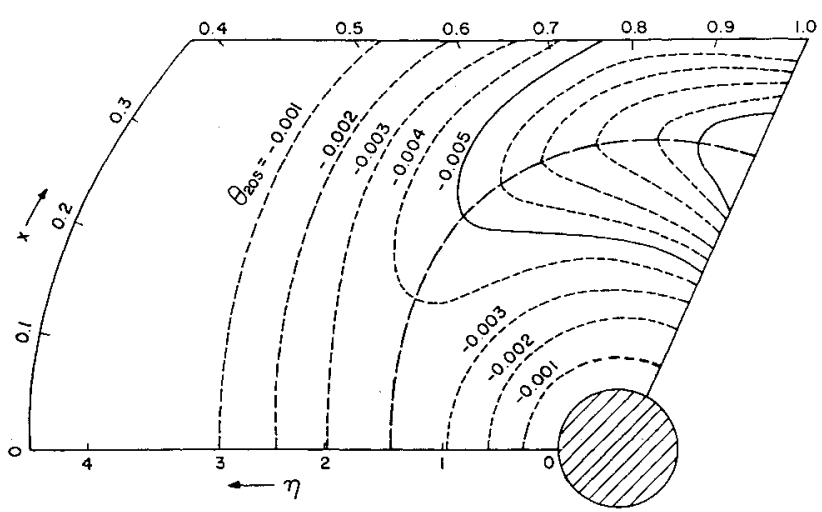

Fig. 4a Distribution of the steady-secondary component of temperature or concentration produced by flow past a circular cylinder with fluctuating circulations of amplitude, $\epsilon u_{1}=\epsilon$ for a fluid having $\operatorname{Pr}=1$, or $S c=1$, at quasisteady state $(\omega \rightarrow 0)$.

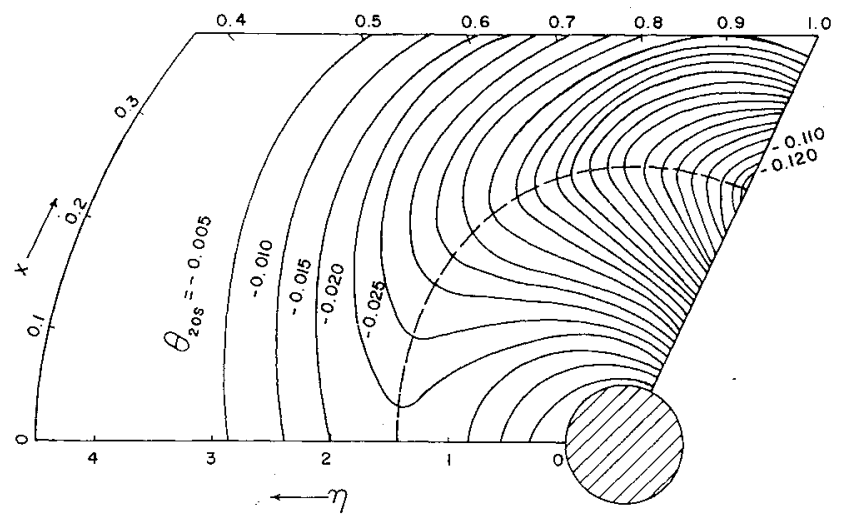

Fig. $4 \mathrm{~b}$ Distribution of the steady-secondary component of temperature or concentration produced by flow past a circular cylinder with fluctuating circulation of amplitude $\epsilon u_{1}(x)=\epsilon\left(1+x^{2}+x^{4}\right)$ for a fluid having $\operatorname{Pr}=1$ or $S c=1$, at quasi-steady state $(\omega \rightarrow 0)$.

location of the vortex from infinity toward the cylinder. The effect of the uniform blowing on the velocity response is to increase the amplitude and to decrease the phase angle, although not significantly. For temperature response, the uniform blowing tends to decrease both the amplitude and phase lag. The uniform suction of the boundary layer affects the response in the opposite manner. The Prandtl number is related only to the thermal boundary layer in forced convection. An increase in the Prandtl number produces a steeper and larger amplitude profile, a smaller phase lag, and a thinner thermal boundary layer.

The second-order solutions, as demonstrated in the analysis, consist of the secondary-steady and periodic components. The steady components represent permanent alterations in the velocity and temperature profiles in the laminar boundary layer caused by the superposition of the alternating vortex. This provides a steady-secondary flow, or streaming, and permanently alters the wall shear stress and heat-transfer rate. Figures 3 and 4 present the streamline $\psi_{20 s}$ and temperature distribution $\theta_{20 s}$ of the steady-secondary components under the quasi-steady condition $\omega \rightarrow 0$. In order to demonstrate the effect of the location of the alternating vortex on the secondary streaming, two vortices are studied. One is placed at infinity and the other is shifted toward the cylinder. Their amplitudes $\epsilon U_{1}$ may be expressed by $\epsilon$ and $\epsilon\left(1+x^{2}+x^{4}\right)$, respectively. A comparison of Figs. $3 a$ and $3 b$ shows that the streamline patterns are essentially similar, except that the alternating vortex at infinity causes weaker steady-secondary

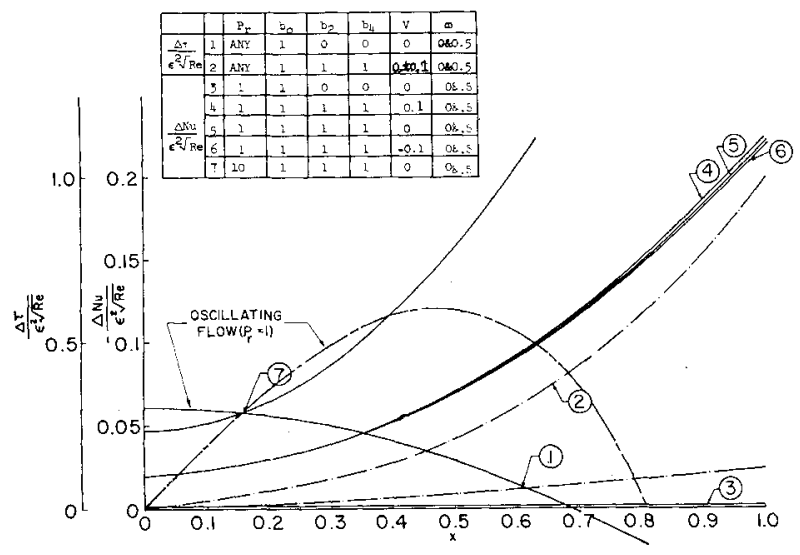

Fig. 5 Effects of fluctuating circulation on the local wall shear stress and Nusselt number for flow past a circular cylinder.

flow in the boundary layer than the other. These streamline patterns are, in general, similar to the one produced by the oscillating flow for which $U_{0}(x)=U_{1}(x)$ (Ref. 21) except in the downstream region. The latter case produces a separation in the secondary streamline at $x \cong 0.8$, which corresponds to an angle of about $90^{\circ}$, where according to the potential flow theory its oscillatory boundary layer is about to separate from the cylinder surface. ${ }^{3}$ This flow oscillation causes an increase in the skin friction from that corresponding to steady flow. The result is given in Fig. 5 for comparison with the present case. A superposition of the uniform blowing tends to delay the separation of the steady-secondary motion. In the present case, the secondary streamlines run closer to the cylinder surface in the downstream direction. The result is that the fluid particles are accelerated along the surface. In other words, the forced convection velocities are increased along the cylinder surface until the point of boundary-layer separation is reached. This causes a continuous increase in the permanent alteration in the skin friction $\Delta \tau$ along $x$ as indicated in Fig. 5. An increase in the fluctuation frequency tends to enhance the secondary flow but its effect is very insignificant (the maximum difference in the order of magnitude between $\psi_{20 s}$ and $\omega^{2} \psi_{22 s}$ is about 20 to 1 for the dimensionless frequency $\omega$ of 0.5). An investigation of Figs. 4 reveals that the steady-secondary component of temperature is negative in the entire oscillatory boundary layer up to the separation point. As the alternating vortex moves from a position at infinity toward the cylinder, one observes: 1) the over-all distribution pattern remains unchanged, 2) the magnitude in $\theta_{20 s}$ is increased, and 3) the isothermal lines in the immediate vicinity of the cylinder surface shift closer toward the surface in the downstream direction. The result is that the temperature gradient at the surface, which is negative in sign, is increased along the surface. This causes a continuous decrease in the permanent alteration in the heat-transfer rate along the surface, as illustrated in Fig. 5. As for the oscillating flow case, ${ }^{21}$ the isothermal lines are pushed away from the surface in the downstream direction due to the steady-secondary flow. A zero isothermal line intersects orthogonally with the cylinder surface at zero $\theta_{20 s}$ at a location between the forward stagnation point and the separation point of the secondary streamline. It is at the intersection point where $\theta_{20 s}$ changes from a negative value upstream to a positive value downstream until the steady-secondary flow separates. This indicates, as shown in Fig. 5, that the steady-secondary flow resulting from the oscillating flow causes a reduction in the local Nusselt number upstream and an increase downstream from that corresponding to steady forced convection. As the Prandtl number increases from 1 to 10 , this intersection point shifts forward from $x=0.7$ to $x=0.42$. For the fluctuating circulation case, the permanent alterations in the skin friction and heat-transfer 
rate as expressed by Eq. (19) are graphically illustrated in Fig. 5. It is shown that the steady-secondary flow resulting from the superposition of an alternating vortex causes an increase in the local skin friction and a reduction in the local heat-transfer rate from those corresponding to steady forced convection. These alterations, however, are very small and are detected from the analysis only when solutions are obtained to at least the second-order approximation beyond the solution for the steady forced convection problem. In general, the alterations are magnified along the surface from the forward stagnation point. A shift in the location of the alternating vortex from infinity toward the cylinder surface, the superposition of the uniform blowing, and an increase in the fluctuation frequency may contribute to an increase in the alterations. It is obvious that the effect of the frequency is very insignificant. This indicates that, in the range of small frequency for which this analysis applies, the quasi-steady solutions may predict qualitatively the effects of fluctuating circulations in the response of the unsteady boundary layer and the permanent changes in the wall shear stress and heattransfer rate.

\section{References}

${ }^{1}$ Lighthill, M. J., "The response of laminar skin friction and heat transfer to fluctuations in the stream velocity," Proc. Roy. Soc. (London) A224, 1-23 (1954).

${ }^{2}$ Lin, C. C., "Motion in the boundary-layer with a rapidly oscillating external flow," Proc. 9th Intern. Cong. Appl. Mech. 4, 155-167 (1956).

${ }^{3}$ Hori, E., "Unsteady boundary layers," Part I, Bull. Japan. Soc. Mech. Eng. 4, no. 16, 664 (1961); Part II 5, no. 17, 57 (1962); Part III 5, no. 17, 64 (1962); also Part IV, Trans. Japan. Soc. Mech. Eng. 27, 1731 (1962).

${ }^{4}$ Stuart, J. T., "A solution of the Navier-Stokes and energy equations illustrating the response of skin friction and temperature of an infinite plate thermometer to fluctuations in the stream velocity," Proc. Roy. Soc. (London) A231, 116-130 (1955).

${ }^{5}$ Illingworth, C. R., "The effects of a sound wave on the compressive boundary-layer on a flat plate," J. Fluid Mech. 3, 471-493 (1958)

6 Jackson, T. W., Purdy, K. R., and Oliver, C. C., "The effects of resonant acoustic vibrations on the Nusselt numbers for a constant temperature horizontal tube," 1961 Intern. Heat Transfer Conf. II, 483-489 (1961).

${ }^{7}$ Fand, R. M. and Kaye, J., "The influence of vertical vibrations on heat transfer by free convection from a horizontal cylinder," 1961 Intern. Heat Transfer Conf. II, 490-498 (1961).

${ }^{8}$ Bayley, F. J., Edwards, P. A., and Singh, P. P., "The effects of flow pulsations on heat transfer by forced convection from a flat plate," 1961 Intern. Heat Transfer Conf. II, 499-509 (1961).

${ }^{2}$ Kestin, J., Maeder, P. F., and Sogin, H. H., "The influence of turbulence on the transfer of heat to cylinders near the stagnation point," Z. Angew. Math. Phys. 12, 115 (1961).

10 Kestin, J., Maeder, P. F., and Wang, H. E., "Influence of turbulence on the transfer of heat from plates with and without a pressure gradient," 1961 Internal Heat Transfer Conf. II, 432 (1961).

${ }_{11}$ Kestin, J., Maeder, P. F., and Wang, H. E., "On boundary layers associated with oscillating streams," Appl. Sci. Res. A10, 1 (1960)

12 Sehoenhals, R. J. and Clark, J. A., "Laminar free convection boundary layer perturbations due to transverse wall vibration," J. Heat Transfer 84, 174 (1962).

${ }^{13}$ Blankenship, V. D. and Clark, J. A., "Effects of oscillation on free convection from a vertical finite plate," J. Heat Transfer 86, 149-158 (1964).

${ }^{14}$ Blankenship, V. D. and Clark, J. A., "Experimental effects of transverse oscillations on free convection of a vertical, finite plate," J. Heat Transfer 86, 159-165 (1964).

${ }^{15}$ Blankenship, V. D. and Clark, J. A., "Laminar free convection from a vertical, infinite plate subjected to transverse oscillation," ASME Paper 64-APM-43; also J. Appl. Mech. (to be published).

${ }_{16}$ Eshghy, S., "The effect of longitudinal oscillations on fluid flow and heat transfer from vertical surfaces in free convection," Ph.D. Thesis, Dept. of Mechanical Engineering, Univ. of Michigan (1963)

${ }_{17} \mathrm{Na}, \mathrm{T}$. Y., "Influence of localized oscillations on laminar flow over a flat plate," Ph.D. Thesis in Mechanical Engineering, Univ. of Michigan (1964).

${ }_{18}$ Schlichting, H., Boundary Layer Theory (McGraw-Hill Book Co., Inc., New York, 1960), 4th ed

${ }^{19}$ Rosenhead, L., Laminar Boundary Layer (Oxford University Press, Oxford, England, 1963).

${ }^{20}$ Frössling, N., "Evaporation, heat transfer, and velocity distribution in two dimensional and rotationally symmetrical laminar boundary-layer flow," NACA TM 1432 (1958).

${ }^{21}$ Yang, W. J. and Clark, J. A., "Influence of flow and rotational oscillations on the mechanics of two-dimensional laminar boundary-layer flow past cylinders including uniform suction or bowing," TR of ORA Project 05065, Univ. of Michigan (June 1964)

${ }^{22}$ McGregor, D. M., "An experimental investigation of the oscillating pressures on a circular cylinder in a fluid stream," Univ. of Toronto, Institute of Aerophysics, TN 14 (1957).

${ }^{23}$ Frössling, N., "Problems of heat transfer across laminar boundary layers," Theory and Fundamental Research in Heat Transfer, edited by J. A. Clark (Pergamon Press, New York, $1963)$. 